\title{
Variação Temporal no Desempenho em Testes de Memória em Pacientes com Doença Vascular Cerebral
}

\section{Temporal Variation in Memory Tests Performance in Cerebral Vascular Disease Patients}

\author{
Tania Fernandes Campos*, Damião Ernane de Souza, Carolina Dutra \\ Gomes Pinheiro \& Alexandre Augusto de Lara Menezes \\ Universidade Federal de Rio Grande do Norte, Natal, Brasil
}

\begin{abstract}
Resumo
Buscando adequar a avaliação neuropsicológica à organização temporal do organismo humano, avaliou-se o desempenho em testes de memória em 12 pacientes pós Doença Vascular Cerebral e 12 indivíduos controle, de ambos os sexos, com idade de 45 a 65 anos. Foram aplicados dois testes de memória com estímulos visuais (figuras) e dois com estímulos verbais (palavras), em 3 dias consecutivos por semana, às 08:00, 10:00 e 12:00 $\mathrm{h}$ na primeira semana e às 14:00 e 16:00 h na seguinte. Os pacientes apresentaram menor número de acertos do que os indivíduos controle em todos os testes aplicados $(p<0,05)$. A sensibilidade foi maior às 14 hoomin para o teste de evocação livre e às 16 hoomin para os testes de reconhecimento. Diante dos resultados, conclui-se que a avaliação neuropsicológica deva ser realizada preferencialmente à tarde, tanto na avaliação inicial quanto nas reavaliações.

Palavras-chave: Memória; doença vascular cerebral; variação temporal.
\end{abstract}

\begin{abstract}
This study intended to investigate the performance in memory tests in order to adequate the neuropsychological evaluation to the temporal order of the human organism. Twelve cerebral vascular accident patients and 12 controls, of both sexes and 45-65 years old were studied. Two memory tests with visual stimuli (pictures) and two with verbal stimuli (words) were applied three times a day (08:00, 10:00 and 12:00 h) during the first week and twice a day (14:00 and 16:00 h) in the second week, during three consecutive days in two consecutive weeks. The patients showed lower scores than control subjects in all applied tests $(p<0,05)$. The greater test sensitivity was at 14:00 $\mathrm{h}$ for the free recall test and at 16:00 $\mathrm{h}$ for recognition tests. According to these results, it is concluded that neuropsychological evaluations should be conducted preferably in the afternoon, as well for the first evaluation as for the re-evaluations.

Keywords: Memory; cerebral vascular disease; temporal variation.
\end{abstract}

A variação diurna do desempenho em testes neuropsicológicos, que avaliam a atenção, a memória e funções executivas, tem sido cada vez mais evidenciada. Diferentes perfis de variação temporal podem ser encontrados, conforme a função ou comportamento avaliado. Isto é decorrente da atuação de um sistema de múltiplos osciladores e da influência de pistas temporais do ambiente como o ciclo claro/escuro (dia/noite) e fatores sociais como horários escolares, de alimentação e de trabalho (Dijk, \& Von Schantz, 2005; Honma, Hashimoto, Nakao \& Honma, 2003).

Avaliando tarefas cognitivas que envolvem a aritmética mental e a memória de curto-prazo foi observado maior desempenho durante a manhã (Monk, 1994). Por outro lado, foi verificado que a memória de longo-prazo flutua de forma diferente, sendo a retenção de informações fornecidas à

\footnotetext{
* Endereço para correspondência: Centro de Ciências da Saúde, Departamento de Fisioterapia, Rua General Gustavo Cordeiro de Farias, s/n, Petrópolis, Natal, RN, 59010-180. E-mail: taniacampos@ufrnet.br
}

tarde melhor do que pela manhã (Folkard, Monk, Bradbury \& Rosenthall, 1977). Na avaliação da memória implícita, encontrou-se maior desempenho pela manhã para indivíduos vespertinos e à tarde para matutinos (May, Hasher \& Foong, 2005).

As evidências da influência do sistema de temporização circadiana (cerca de $24 \mathrm{~h}$ ) dos indivíduos sobre o seu desempenho nos testes, tem embasado as mudanças nos horários escolares (Andrade \& Menna-Barreto, 1996) e adaptações nos esquemas de trabalho (Rotemberg, Portela, Marcondes, Moreno \& Nascimento, 2001) a fim de otimizar o desempenho em tarefas escolares e nas atividades ocupacionais.

$\mathrm{Na}$ área clínica, as investigações sobre a variação temporal de funções e comportamentos têm contribuído para aumentar a precisão diagnóstica e a eficácia da terapia medicamentosa, diminuindo os efeitos colaterais (Klerman, 2005; Smolensk, 1998), além de possibilitar a caracterização de distúrbios circadianos em pacientes com patologias neurológicas como a Doença de Alzheimer e a Doença 
Vascular Cerebral (Bonanni et al., 2005; Campos et al., 2005; Harper et al., 2004).

A Doença Vascular Cerebral (DVC) é uma patologia muito freqüente e causa importante de morbidade e mortalidade (Nyenhuis \& Gorelick, 1998). Na prática clínica, o termo DVC é utilizado para especificar o déficit neurológico decorrente da lesão vascular e representa um grupo de doenças com etiologias e manifestações clínicas variadas. A localização da lesão está relacionada com alterações neurológicas específicas como déficits motores, sensitivos e cognitivos, incluindo comprometimentos da linguagem, memória e funções executivas. Segundo Allegri, López e Carrá (1993), pacientes com lesão no hemisfério cerebral esquerdo exibem déficit na memória para estímulos verbais, enquanto que o comprometimento da memória para estímulos visuais ocorre em pacientes com lesão cerebral à direita.

Os testes neuropsicológicos têm uma aplicação abrangente e são comumente utilizados para a diagnóstico de funções cognitivas comprometidas em diferentes patologias como Doença Vascular Cerebral, Doença de Parkinson e Doença de Alzheimer (Kuslansky et al., 2004), muitas vezes permitindo estabelecer correlações entre a localização da lesão cerebral e a presença dos déficits cognitivos (Garrett et al., 2004; Lindeboom \& Weinstein 2004; Sachdev et al., 2004), entre outras indicações. As baterias de testes freqüentemente empregadas são as Escalas Wechsler, de inteligência (WAIS-III) e de memória (WMS-III), bastante utilizadas na avaliação das capacidades cognitivas de crianças, adolescentes e adultos; a bateria CERAD (Consortium to Establish a Registry for Alzheimer's Disease), capaz de sugerir se o paciente está apresentando declínio cognitivo associado à idade, detectar transtorno cognitivo leve ou identificar os estágios da Doença de Alzheimer e o Mini-exame do Estado Mental (MEEM), um exame de screening das funções cognitivas, que analisa a memória, orientação temporal e espacial, atenção e cálculo, linguagem e capacidade construtiva visual. Dentre as baterias de testes aplicadas no Brasil existem o Teste de Discriminação de Listas de palavras e figuras, utilizado na avaliação de pacientes com lesões ou disfunções do lobo frontal (Haase, Lacerda, Wood, Daker \& Peixoto, 2001) e a bateria de testes neuropsicológicos computadorizados (Charchat, Nitrini, Caramelli \& Sameshima, 2001), constituída de testes para avaliar a memória episódica (com figuras de faces, desenhos e palavras), de curto-prazo e implícita, que foi utilizada para identificar o comprometimento da memória durante o estágio inicial da Doença de Alzheimer.

$\mathrm{Na}$ abordagem clínica, os testes da avaliação neuropsicológica são indiferentemente aplicados pela manhã ou à tarde. Durante o tratamento, o indivíduo é reavaliado usando o mesmo procedimento para determinar sua evolução e prognóstico. No entanto, analisar o desempenho em testes de memória sem considerar sua variação em função da hora poderá resultar em valores falsos positivos ou falsos negativos. A literatura não mostra estudos abordando a variação temporal no desempenho em testes de memória nos pacientes pós-DVC, tornando desconhecido o grau de precisão desses testes tanto na avaliação quanto nas reavaliações do comprometimento apresentado pelos pacientes.

\section{Objetivo}

O presente estudo teve como objetivo identificar a variação em função da hora no desempenho em testes de memória com estímulos visuais e verbais, em pacientes com Doença Vascular Cerebral, considerando o lado cerebral afetado e o funcionamento do sistema de temporização circadiana (STC). Pretendeu-se sugerir um horário mais apropriado para a realização de testes de memória, a fim de adequar a avaliação neuropsicológica à organização temporal biológica.

\section{Sujeitos e Método}

\section{Sujeitos}

A definição da amostra foi realizada através de um levantamento dos pacientes registrados no Serviço de Fisioterapia do Hospital Universitário Onofre Lopes da Universidade Federal do Rio Grande do Norte (UFRN), de janeiro a dezembro de 2002. A partir desse levantamento, foram registrados 54 pacientes e as informações sobre eles foram obtidas através de prontuários, cartas e por telefone. Desses pacientes, foram excluídos os que estavam fora dos critérios de inclusão e a amostra foi constituída por $23 \%$ dos pacientes atendidos naquele ano.

A faixa etária definida para o estudo foi de 45 a 65 anos, inicialmente devido à grande incidência de DVC nessas décadas de vida, adicionalmente, porque a inclusão de indivíduos abaixo de 45 anos implicaria na necessidade de avaliar a influência do ciclo hormonal no desempenho dos testes de memória nas mulheres. Também não foram incluídos os indivíduos acima de 65 anos, porque poderiam apresentar um declínio da memória devido ao envelhecimento. Foram incluídos pacientes com baixa escolaridade por ser essa a demanda de pacientes no Serviço de Fisioterapia. Os indivíduos do grupo controle como eram funcionários públicos, apresentavam menor idade e maior tempo de escolaridade do que os pacientes.

A amostra foi constituída por 24 sujeitos, de ambos os sexos, com idade entre 45 e 65 anos. O grupo de pacientes foi formado por 6 homens e 6 mulheres, com lesão cerebral unilateral (6 à direita e 6 à esquerda), de ambos os tipos de DVC (isquêmico e hemorrágico). Os critérios de exclusão para o grupo de pacientes foram: lesão cerebral extensa, bilateral e recorrente, transtornos cognitivos graves, indivíduos analfabetos ou com curso superior, pacientes no estágio agudo da DVC e que faziam uso de medicamentos com ação no sistema nervoso central como os antidepressivos ou neurolépticos. O grupo controle foi constituído por 6 homens e 6 mulheres, funcionários do mesmo hospital, que não apresentavam qualquer tipo de patologia.

\section{Método}

O estudo foi do tipo duplo cego, onde nem os voluntários e nem os examinadores tinham conhecimento a respeito dos 
objetivos da pesquisa, tendo sido aprovado pelo Comitê de Ética em Pesquisa da UFRN. Os participantes receberam explicações sobre os procedimentos dos testes e deram o seu consentimento por escrito.

\section{Avaliação Clínica}

Os pacientes foram submetidos a uma avaliação neurológica através da escala NIHSS (National Institute of Health Stroke Scale) (Goldstein, Bertels \& Davis, 1989). A pontuação de 0 a 6 indicava comprometimento leve, de 7 a 16, moderado, e grave de 17 a 30. Foram identificados o tempo de seqüela e a dominância manual. A localização da lesão cerebral foi determinada através de tomografia computadorizada. Para os indivíduos controle foi utilizado o Índice de co-morbidades - CIRS (Cumulative Illness Rating Scale) para avaliar a existência de patologias em seis sistemas orgânicos: cardiovascular/respiratório, gastrointestinal, genitourinário, músculo-esquelético e tegumentar, neuropsicológico e sistema geral/endócrino (Xavier, Ferraz, Pertollucci, Poyares, \& Moriguchi, 2001).

Foi empregada uma bateria de testes de memória com figuras e palavras, onde os procedimentos foram retirados do CERAD (Consortium to Establish a Registry for Alzheimer's Disease), na versão validada para o Brasil (Bertolucci et al, 2001). A partir de uma relação de 400 palavras (Pompéia \& Bueno 1998; Pompéia, Miranda \& Bueno, 2001) foram formadas listas de figuras e palavras que representavam substantivos concretos (objetos e animais) da vida cotidiana. Cada figura foi impressa em preto e branco em um cartão de $7 \times 7 \mathrm{~cm}$. As listas de palavras foram gravadas em estúdio especializado e arquivadas em compact-disc (CD). Essa bateria de testes teve a finalidade de avaliar o desempenho da memória episódica por estimulação visual (figuras) e verbal (palavras), utilizando evocação livre e por reconhecimento.

\section{Esquema de Aplicação dos Testes}

As sessões de testes com figuras e palavras foram realizadas durante duas semanas consecutivas, três dias por semana, de $3^{\mathrm{a}}$ a $5^{\mathrm{a}}$ feira. Na primeira semana as sessões de teste foram realizadas às 08:00, 10:00 e 12:00 h e na semana seguinte às 14:00 e 16:00 h. No total, cada indivíduo foi submetido a 15 sessões de teste. O planejamento foi realizado dessa forma para reduzir as alterações na expressão cotidiana da organização temporal biológica do indivíduo. Após o término dos testes, os participantes puderam retornar para suas residências, permanecendo sincronizados ao ciclo claro/escuro natural e a outras pistas temporais. Os testes foram realizados individualmente em local confortável, com luminosidade adequada e com o mínimo de ruído ambiente. Foram utilizadas seqüências diferentes de figuras e palavras em cada repetição dos testes nos diversos horários. Durante a etapa de apresentação dos estímulos, os participantes não eram avisados de que sua memória seria testada, como também não receberam treinamento anterior. As instruções para a realização dos testes foram padronizadas e todos os participantes foram submetidos às mesmas seqüências. Participaram dessa etapa quatro examinadores adequadamente treinados que realizaram as sessões de teste em regime de rodízio. Foram aplicados os testes: memória episódica com reconhecimento imediato de figuras (FIGIM) e reconhecimento tardio (FIGTAR), memória episódica com evocação livre imediata de palavras (PALIM) e reconhecimento tardio (PALTAR), com duração de 3 a 5 minutos cada um (os procedimentos dos testes encontramse em anexo).

Para avaliar se a lesão neurológica havia afetado o funcionamento do sistema de temporização circadiana foram investigadas as características do ciclo vigília/sono e a variação diurna da temperatura oral. Foi utilizado o Questionário de Horne e Östberg na versão traduzida e validada para o Brasil (Benedito-Silva, Menna-Barreto, Marques \& Tenreiro, 1990) a fim de determinar o caráter de matutinidade e vespertinidade e definir o cronotipo dos indivíduos. O diário de sono foi utilizado para registrar os horários de dormir e acordar, antes e durante a semana de realização dos testes de memória. A temperatura oral de cada participante foi medida antes de cada sessão de teste, com termômetro digital colocado na região sub-lingual durante 2 minutos.

\section{Análise dos Dados}

As diferenças entre pacientes e grupo controle quanto às variáveis demográficas, neurológicas, do ciclo vigília-sono (CVS) e desempenho nos testes de memória foram verificadas através do teste de Mann-Whitney. Verificouse o perfil da variação diurna da temperatura oral e analisou-se o efeito da hora através do teste de Friedman. A análise post hoc foi realizada através do teste de NewmanKeuls. Para a análise citada utilizou-se o programa Statistica 5.5, atribuindo-se o nível de significância de 5\% para todos os testes estatísticos.

Para verificar a variação no poder discriminativo de cada teste na avaliação do desempenho da memória de acordo com a hora, os dados foram analisados por meio dos programas GraphPad Instat 3.0 e SPSS 10.0 (Statistical Package for the Social Science). Foram avaliadas tabelas $2 \times 2$ considerando cada teste em cada horário contra o respectivo padrãoouro. No padrão-ouro, foi classificado como caso positivo o indivíduo que apresentava um número de acertos igual ou inferior ao ponto de corte em todos os horários de realização dos testes e nos quatro testes realizados. O ponto de corte de cada teste de memória foi estimado através do percentil 25 da distribuição de valores da amostra estudada. Por fim, foram estabelecidos a sensibilidade, a especificidade, o valor preditivo positivo, o valor preditivo negativo e o intervalo de confiança a $95 \%$. Os valores foram apresentados na curva ROC (Receiver Operating Curves) considerando o tipo de teste e a hora de maior poder discriminativo.

\section{Resultados}

A média de idade dos pacientes (60 \pm 6 anos; média \pm desvio padrão) foi maior do que a do grupo controle (53 \pm 6 anos) $(\mathrm{U}=29,0 ; p=0,0105)$, enquanto que a escolaridade 
foi menor nos pacientes ( $4 \pm 1$ anos) do que no grupo controle $(9 \pm 2$ anos $)(\mathrm{U}=3,0 ; p=0,0001)$. Todos os pacientes apresentavam dominância lateral direita antes da DVC e após a lesão, 6 deles passaram a realizar suas atividades diárias com o membro superior esquerdo.

Pela tomografia computadorizada foi verificado que 7 pacientes apresentavam lesões múltiplas (unilaterais) localizadas nos lobos frontal, pariental e temporal, e 5 lesões únicas, sendo 3 no lobo parietal e 2 nos núcleos da base. Os pacientes avaliados apresentavam comprometimento da força muscular e da coordenação motora de membros superior e inferior, além de déficits de sensibilidade, linguagem e esquema corporal, com escores no NIHSS variando de 3 a $13(7 \pm 3)$ mostrando em média um comprometimento neurológico moderado, confirmando as lesões cerebrais encontradas. Não foi encontrada diferença nos escores do NIHSS entre os pacientes com lesão cerebral à direita e à esquerda ( $\mathrm{U}=11,0 ; p=0,2623)$. O tempo de seqüela médio dos pacientes foi de $20 \pm 10$ meses, sem diferença de acordo com o lado da lesão $(\mathrm{U}=9,0 ; p=0,1495)$. Dos pacientes avaliados $67 \%$ faziam uso de medicamentos antihipertensivos, $42 \%$ de antidiabéticos, 33\% de antiagregantes plaquetários e $8 \%$ para insuficiência cardíaca, os quais não tinham ação no sistema nervoso central e nem influenciavam o desempenho nos testes de memória.

A análise do desempenho nos testes de memória mostrou que os pacientes acertaram menos que os indivíduos controle nos testes FIGIM (U=12794,0; $p=0,0001)$, FIGTAR $(\mathrm{U}=10983,5 ; p=0,0001), \operatorname{PALIM}(\mathrm{U}=9295,5 ; p=0,0001)$ e PALTAR (U=9270,0; $p=0,0037)$ (Figura 1). Os pacientes com lesão à direita apresentaram menor número de acertos nos testes FIGIM (U=3004,0; $p=0,0028)$ e FIGTAR $(\mathrm{U}=2403,0 ; p=0,0001)$, enquanto que os pacientes com lesão à esquerda tiveram mais acertos no teste PALTAR $(\mathrm{U}=2944,0 ; p=0,0016)$.

A análise dos dados por hora foi realizada através de múltiplos testes de Mann-Whitney. Ocorreram diferenças entre indivíduos controle e pacientes nos testes PALIM e PALTAR em todos os horários de realização dos testes. Entretanto, no teste de FIGIM não se observou diferença às 08:00 h, 10:00 h e 16:00 h e no teste FIGTAR às 12:00 h (Tabela 1).

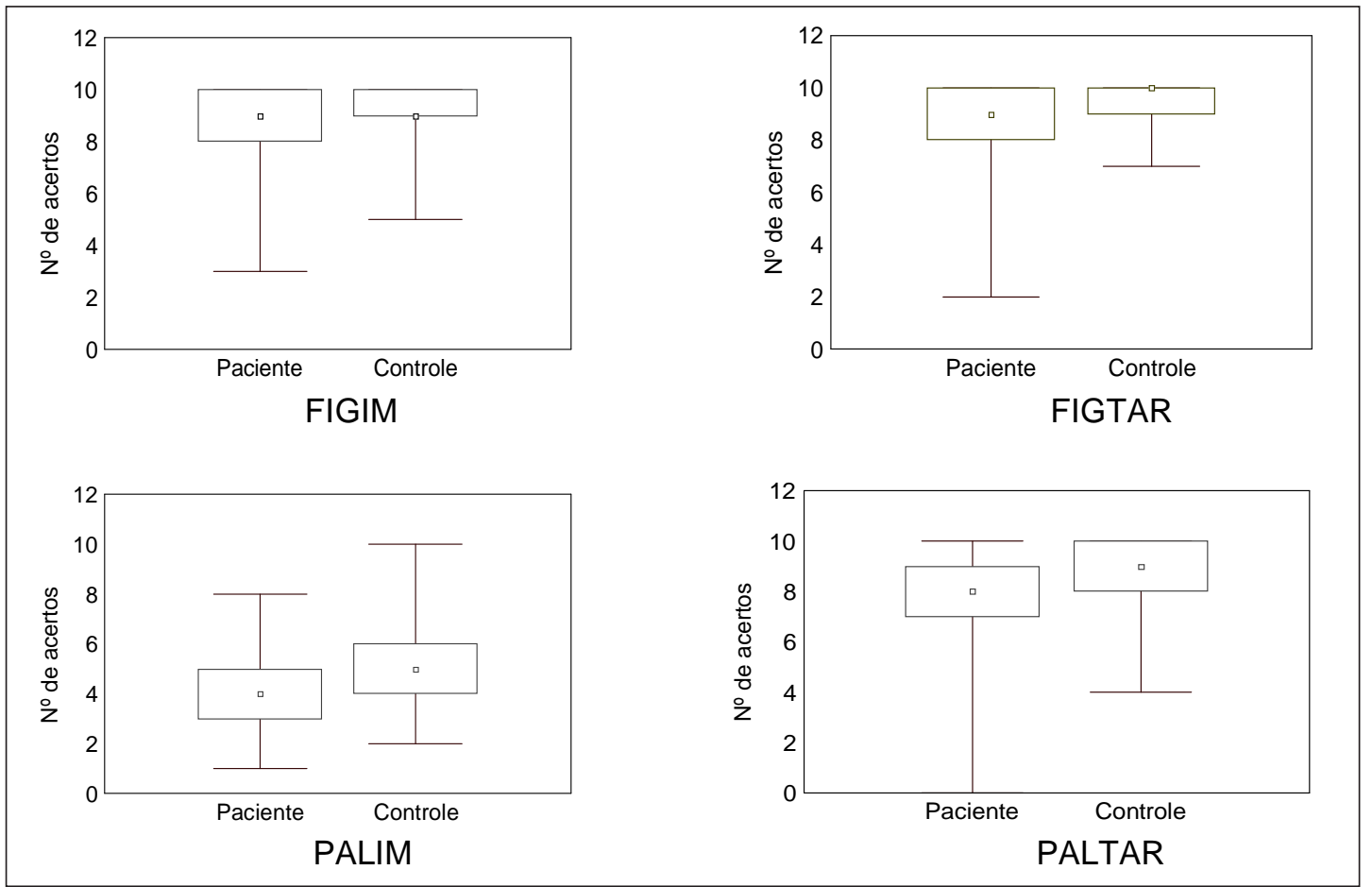

Figura 1. Número de acertos (mediana, quartis, mínimo-máximo) nos testes de memória obtidos pelos indivíduos controle e pacientes.

Tabela 1

Comparação do Número de Acertos nos Testes de Memória de Pacientes e Indivíduos Controle de acordo com o Horário de Aplicação dos Testes

\begin{tabular}{cccccc}
\hline & \multicolumn{5}{c}{ Horário de Aplicação do Teste } \\
\cline { 2 - 6 } & $08: 00$ & $10: 00$ & $12: 00$ & $14: 00$ & $16: 00$ \\
\hline FIGIM & $583,5(0,468)$ & $486,5(0,069)$ & $445,5(0,030)$ & $473,5(0,049)$ & $531,0(0,188)$ \\
FIGTAR & $437,0(0,017)$ & $340,5(\mathrm{O}, 001)$ & $524,5(0,164)$ & $466,5(0,041)$ & $429,0(0,014)$ \\
PALIM & $306,0(0,001)$ & $391,0(0,004)$ & $348,0(0,001)$ & $392,0(0,004)$ & $437,0(0,017)$ \\
PALTAR & $364,0(0,001)$ & $332,5(\mathrm{O}, 001)$ & $416,0(0,009)$ & $352,0(0,001)$ & $384,5(0,003)$ \\
\hline
\end{tabular}

Nota. Os resultados estão apresentados no formato valor de $\mathrm{U}$ (valor de $p$ ). 
A Tabela 2 apresenta a sensibilidade, os valores preditivos positivo e negativo dos testes variando de acordo com a hora, sendo maiores às 16:00 h para os testes FIGIM e FIGTAR e às 14:00 h para o teste PALIM. A especificidade variou apenas no teste FIGIM. No teste PALTAR, o maior valor da sensibilidade foi 0,71 , observado às 10:00 e 16:00 h, mas a especificidade foi muito baixa $(0,20)$ em todos os horários. $\mathrm{O}$ valor preditivo positivo variou de 0,45 a 0,55 e o valor preditivo negativo de 0,21 a 0,33. Analisando-se a curva ROC (Figura 2), verifica-se que os testes FIGIM e FIGTAR apresentam maior poder discriminativo do desempenho da memória às 16:00 h. Para o teste PALIM isso ocorreu no horário das 14:00 h, enquanto que para o teste PALTAR não foi possível determinar um horário correspondente, pois os valores calculados ficaram abaixo da linha diagonal do gráfico.
Quanto ao ciclo vigília-sono não foram verificadas diferenças entre pacientes e indivíduos controle na pontuação do cronotipo ( $\mathrm{U}=68,5 ; p=0,8399)$ e de acordo com a localização da lesão nos pacientes ( $\mathrm{U}=10,5$; $p=0,2298)$. Ambos os grupos apresentavam características de matutinidade (preferiam dormir e acordar cedo). De acordo com o diário de sono da semana anterior à aplicação dos testes de memória, os indivíduos controle dormiram mais tarde e acordaram mais cedo; na semana dos testes, não houve diferença entre os grupos quanto à hora de acordar, embora tenha havido quanto à hora de dormir (Tabela 3).

De acordo com o teste de Friedman, não houve variação diurna na temperatura oral dos indivíduos controle $(p=$ 0,2048), enquanto que nos pacientes o valor máximo foi registrado às $14: 00 \mathrm{~h}(p=0,0251)$.

Tabela 2

Variáveis Relativas à Validade dos Testes de Memória Utilizados de Acordo com o Horário de Aplicação do Teste

\begin{tabular}{|c|c|c|c|c|c|c|}
\hline Teste & Hora & Sensibilidade & IC & Especificidade & VPP & VPN \\
\hline \multirow[t]{5}{*}{ FIGIM } & 08:00 & 0,40 & $0,27-0,55$ & 0,35 & 0,62 & 0,18 \\
\hline & $10: 00$ & 0,65 & $0,51-0,78$ & 0,30 & 0,71 & 0,25 \\
\hline & 12:00 & 0,67 & $0,53-0,79$ & 0,30 & 0,71 & 0,26 \\
\hline & $14: 00$ & 0,55 & $0,40-0,69$ & 0,30 & 0,67 & 0,21 \\
\hline & $16: 00$ & 0,73 & $0,58-0,84$ & 0,30 & 0,73 & 0,30 \\
\hline \multirow[t]{5}{*}{ FIGTAR } & 08:00 & 0,56 & $0,39-0,70$ & 0,37 & 0,57 & 0,36 \\
\hline & 10:00 & 0,53 & $0,37-0,68$ & 0,37 & 0,56 & 0,35 \\
\hline & 12:00 & 0,56 & $0,39-0,70$ & 0,37 & 0,57 & 0,36 \\
\hline & $14: 00$ & 0,55 & $0,39-0,70$ & 0,37 & 0,57 & 0,36 \\
\hline & $16: 00$ & 0,81 & $0,66-0,91$ & 0,37 & 0,66 & 0,57 \\
\hline \multirow[t]{5}{*}{ PALIM } & 08:00 & 0,58 & $0,42-0,72$ & 0,41 & 0,59 & 0,40 \\
\hline & 10:00 & 0,46 & $0,31-0,62$ & 0,41 & 0,54 & 0,34 \\
\hline & 12:00 & 0,48 & $0,33-0,64$ & 0,41 & 0,55 & 0,35 \\
\hline & $14: 00$ & 0,83 & $0,69-0,93$ & 0,41 & 0,68 & 0,63 \\
\hline & $16: 00$ & 0,44 & $0,29-0,60$ & 0,41 & 0,52 & 0,33 \\
\hline \multirow[t]{5}{*}{ PALTAR } & 08:00 & 0,59 & $0,43-0,74$ & 0,20 & 0,51 & 0,26 \\
\hline & 10:00 & 0,71 & $0,55-0,84$ & 0,20 & 0,55 & 0,33 \\
\hline & 12:00 & 0,66 & $0,50-0,80$ & 0,20 & 0,53 & 0,30 \\
\hline & $14: 00$ & 0,47 & $0,32-0,63$ & 0,20 & 0,45 & 0,21 \\
\hline & $16: 00$ & 0,71 & $0,55-0,84$ & 0,20 & 0,55 & 0,33 \\
\hline
\end{tabular}

Notas. IC: Intervalo de confiança; VPP: Valor preditivo positivo; VPN: Valor preditivo negativo.

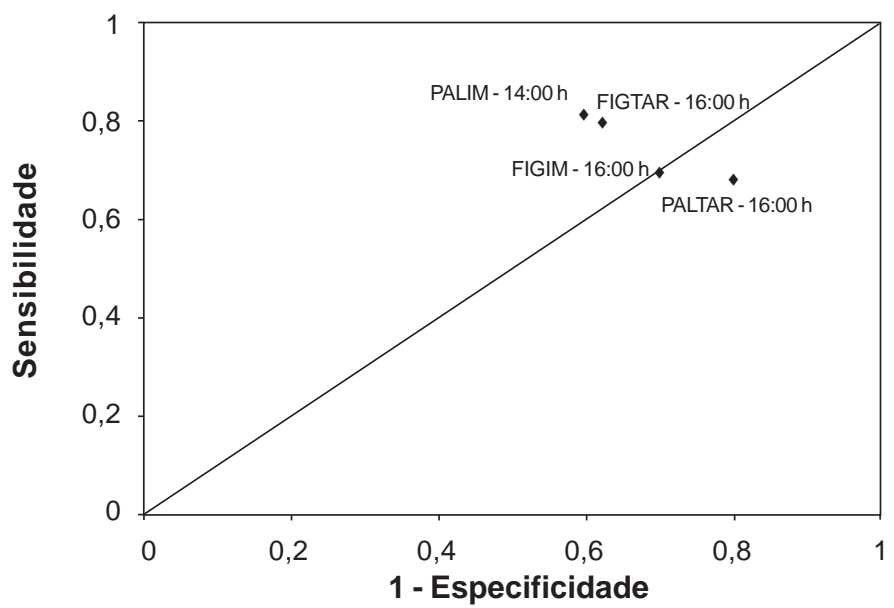

Figura 2. Coordenadas da Curva ROC para os testes utilizados, considerando os horários de maior poder discriminativo. 
Tabela 3

Comparação entre Pacientes e Controle quanto às Características do Ciclo Vigília-Sono

\begin{tabular}{llccc}
\hline & & Paciente & Controle & $\mathrm{p}$ \\
\hline \multirow{2}{*}{ Antes } & Dormir (h:min \pm min) & $21: 25 \pm 17$ & $22: 13 \pm 3$ & 0,0001 \\
& Acordar (h:min \pm min) & $06: 03 \pm 1$ & $05: 50 \pm 2$ & 0,0001 \\
& & & & \\
\multirow{2}{*}{ Durante } & Dormir (h:min \pm min) & $21: 17 \pm 30$ & $22: 14 \pm 9$ & 0,0001 \\
& Acordar (h:min \pm min) & $05: 39 \pm 1$ & $05: 27 \pm 40$ & n.s. \\
\hline
\end{tabular}

Nota. n.s.= não significativo

\section{Discussão}

Além da avaliação clínica, os testes de memória empregados no presente estudo permitiram discriminar o grupo controle do grupo clínico, onde os pacientes tiveram menor número de acertos. É importante salientar que os dois grupos apresentaram maior número de acertos nos testes com figuras do que com palavras, conforme também foi observado em outro estudo clínico (Haase et al., 2001). Isso mostra que houve diferença nos desempenhos dos indivíduos de acordo com o tipo de memória avaliado, a modalidade de estímulo utilizada e o tipo de evocação realizada. De acordo com a literatura, o estímulo visual favorece o processamento da informação (Stenberg, Radeborg \& Hedman, 1995) e o reconhecimento prevalece sobre as outras formas de evocação (Lacerda \& Haase, 2000). Entretanto, ao separar os pacientes de acordo com o lado da lesão cerebral verificou-se que a superioridade do desempenho em testes de figuras foi determinada pelos pacientes com lesão cerebral à esquerda, pois os que tinham lesão à direita, apresentaram menor número de acertos no teste FIGTAR, com o inverso ocorrendo no teste PALTAR, o que pode estar relacionado com a especialização do hemisfério cerebral direito para funções visuo-espaciais e o esquerdo para funções linguísticas e verbais (Allegri et al., 1993).

As diferenças entre os indivíduos controle e os pacientes do desempenho nos testes de memória foram encontradas em alguns horários mas não em outros, sugerindo que o examinador pode, de fato, obter uma informação imprecisa se não levar em consideração a hora de realização do teste. Assim, a avaliação de um indivíduo cujo desempenho está no limite inferior da normalidade realizada no horário de menor desempenho poderá apresentar resultado abaixo da faixa de normalidade, podendo o individuo não afetado ser considerado como caso positivo. Por outro lado, um indivíduo com comprometimento leve avaliado no momento de máximo desempenho poderá mostrar resultado dentro do limite normal e ser considerado como caso negativo.

Os pontos de corte, critério geralmente empregado para diferenciar grupos clínicos (Charchat et al., 2001; Haase et al., 2001), também variaram em função da hora, corroborando a necessidade de considerar as flutuações diurnas no desempenho. No presente estudo, a sensibilidade dos testes foi maior no horário de menor ponto de corte, implicando que nesse horário houve maior freqüência de indivíduos com baixo número de acertos. Os testes de memória foram sensíveis para detectar os casos positivos, ou seja, aqueles que apresentavam desempenho abaixo do ponto de corte, mais do que para identificar os casos negativos, os que tinham desempenho acima do ponto de corte, pois todos os testes apresentaram sensibilidade maior do que especificidade. É possível que dentre os casos positivos sejam encontrados mais pacientes do que indivíduos do grupo controle, em função das diferenças verificadas entre os grupos quanto a idade e escolaridade, os quais são fatores que influenciam significativamente o desempenho em testes de memória (Bertolucci, Brucki, Capacci \& Juliano, 1994; Bertolucci et al., 2001).

Os valores mais altos de sensibilidade foram observados durante a tarde, próximo dos horários do final da atividade e do pico da temperatura, o que poderia estar relacionado com o funcionamento do sistema de temporização circadiana. $\mathrm{O}$ horário no qual a sensibilidade foi maior diferiu apenas no teste PALIM, único dos testes utilizados que realizava a evocação livre, mas o turno não foi diferente dos demais testes. A literatura refere que a relação entre o desempenho em tarefas com processamento mental e a temperatura corporal decorre do fato de que os processos mentais representam reações químicas que dependem do nível de atividade metabólica das células do córtex cerebral, a qual é estimulada pelo aumento da temperatura corporal (Wright, Hull \& Czeisler, 2002). Do ponto de vista cronobiológico, essa relação está traduzida na ordem temporal interna que é conseqüência da manutenção de uma relação estável entre as fases dos ritmos fisiológicos e comportamentais (Wright et al., 2002).

$\mathrm{O}$ valor preditivo positivo dos testes foi maior do que o valor preditivo negativo, indicando maior capacidade dos testes em detectar os pacientes entre os que apresentaram comprometimento da memória, sendo esse achado importante para o diagnóstico diferencial entre patologias neurológicas. Os intervalos de confiança altos, mas diferentes entre os horários, também indicaram que podem ocorrer falhas de diagnóstico no sentido de falso positivo ou falso negativo, dependendo da hora.

Analisando-se a curva ROC, percebe-se que os testes com maior poder diagnóstico foram, em ordem decrescente: PALIM, FIGTAR e FIGIM. Isso indica que os pacientes apresentaram mais dificuldades no teste com evocação livre e menos dificuldades nos testes com estímulos visuais e na recuperação realizada através de reconhecimento. Embora a amostra examinada seja pequena, esses achados devem ser levados em consideração no momento de estabelecer os indicadores clínicos do estágio crônico da DVC. 
A análise do CVS mostrou semelhança entre os dois grupos quanto ao caráter de matutinidade, não havendo diferença quanto à pontuação do cronotipo indicando que a amostra era homogeneamente constituída por indivíduos que preferiam dormir e acordar cedo. A análise do diário de sono apontou que, na semana anterior à realização dos testes de memória, os pacientes dormiam e acordavam mais tarde do que os indivíduos controle, mas sem diferença quanto ao horário de acordar durante as semanas dos testes. Essa mudança pode estar relacionada com o fato dos indivíduos do grupo controle estarem sincronizados ao horário de trabalho, além de indicar, para ambos os grupos, a capacidade do CVS em se organizar de acordo com a situação imposta ao indivíduo, o que traduz a sua flexibilidade.

O ritmo de temperatura corporal é um importante indicador da atuação do sistema de temporização do organismo humano, sendo comprovado o seu relacionamento com ritmos de alerta e desempenho neurocomportamental. Segundo Wright et al. (2002), funções cerebrais como memória operacional, atenção visual e tempo de reação são melhor desempenhadas quando a temperatura está elevada. Os autores verificaram que o aumento da temperatura em $0,15{ }^{\circ} \mathrm{C}$ pode ser suficiente para influenciar o desempenho humano. O presente estudo conseguiu evidências satisfatórias da atuação do STC na sincronização dos pacientes ao ambiente, o que torna mais confiáveis os achados sobre a flutuação do desempenho da memória. Entretanto, deve-se levar em consideração o número reduzido da amostra, sendo possível que réplicas desse trabalho possam levar a resultados diferentes.

A partir dos resultados já encontrados pode-se sugerir algumas condutas a serem adotados para a avaliação de pacientes que apresentam DVC. É recomendável que o examinador realize pelo menos duas sessões de teste com intervalo de mais de duas horas entre elas. Alternativamente, poderá ser estabelecido um horário para a aplicação do teste e acompanhamento da evolução do paciente. Ao levar em conta a influência do STC no desempenho do indivíduo como parte da rotina de exame, ter-se-ia uma melhor definição da condição clínica dos pacientes com DVC.

\section{Referências}

Allegri, R. F., López, L., \& Carrá, A. (1993). Estudio de la lateralidad amnésica episódica en las lesiones isquémicas temporales internas. Revista Neurologica Argentina, 18, 12-18.

Andrade, M. M. M., \& Menna-Barreto, L. (1996). Diurnal variation in oral temperature, sleepiness and performance of high school girls. Biological Rhythm Research, 27, 336-342.

Benedito-Silva, A. A., Menna-Barreto, L., Marques, N., \& Tenreiro, S. (1990). A self-assessment questionnaire to determine morningness-eveningness types in Brazil. In D. K. Hayes, J. E. Pauli \& R. J. Reiter (Eds.), Chronobiology: Its role in clinical medicine, general biology and agriculture (pp. 89-90). New York: Wiley Liss.

Bertolucci, P. H. F., Brucki, S. M. D., Capacci, S. R., \& Juliano, Y. (1994). O mini-exame do estado mental em uma população geral. Impacto da escolaridade. Arquivos de Neuropsiquiatria, 52, 1-7.
Bertolucci, P. H. F., Okamoto, I. H., Brucki, S. M. D., Siviero, M. O., Toniolo Neto, J., \& Ramos, L.R. (2001). Applicability of the CERAD Neuropsycholgical Batery to Brasilian elderly. Arquivos de Neuropsiquiatria, 59, 532-536.

Bonanni, E., Maestri, M., Tognoni, G., Fabbrini, M., Nucciarone, B., Manca, M. L., Gor , S. I., Iudice, A., \& Murri, L. (2005). Daytime sleepiness in mild and moderate Alzheimer's disease and its relationship with cognitive impairment. Journal Sleep Research, 14, 311-317.

Campos, T. F., Diógenes, F. P., França, F. R., Dantas, R. C. S., Araujo, J. F., \& Menezes, A. A. L. (2005). The sleep - wake cycle in the late stage of cerebral vascular accident recovery. Biological Rhythm Research, 36, $109-113$

Charchat, H., Nitrini, R., Caramelli, P., \& Sameshima, K. (2001). Investigação de marcadores clínicos dos estágios iniciais da doença de Alzheimer com testes neuropsicológicos computadorizados. Psicologia: Reflexão e Crítica, 14, 305-316.

Dijk, D., \& Von Schantz, M. (2005). Timing and consolidation of human sleep, wakefulness, and performance by a symphony of oscillators. Journal of Biological Rhythms, 20, 279-290.

Folkard, S., Monk, T. H., Bradbury, R., \& Rosenthall, J. (1977). Time of day effects in school children's immediate and delayed recall of meaningful material. British Journal of Psychology, $68,45-50$.

Garrett, K. D., Browndyke, J. N., Whelihan, W., Paul, H. R., DiCarlo, M., Moser, D. J., Cohen, R. A., \& Ott, B. R. (2004). The neuropsychological profile of vascular cognitive impairment - no dementia: Comparisons to patients at risk for cerebrovascular disease and vascular dementia. Archives of Clinical Neuropsychology, 19, 745-757.

Goldstein, L. B., Bertels, C., \& Davis, J. N. (1989). Interrater reliability of the NIH stroke scale. Archives of Neurology, 46, 660-662.

Haase, V. G., Lacerda, S. S., Wood, G. M. O., Daker, M. V., \& Peixoto, M. A. L. (2001). Estudos clínicos iniciais com o teste de discriminação de listas (TDL-UFMG). Psicologia: Reflexão $e$ Crítica, 14, 289-304.

Harper D. G., Stopa, E. G., McKee, A. C., Satlin, A., Fish, D., \& Volicer, L. (2004). Dementia severity and Lewy bodies affect circadian rhythms in Alzheimer disease. Neurobiology of Aging, 25, 771-781.

Honma, K., Hashimoto, S., Nakao, M. \& Honma, S. (2003). Period and phase adjustments of human circadian rhythms in the real world. Journal of Biological Rhythms, 18, 261-270.

Klerman, E. B. (2005). Clinical aspects of human circadian rhythms. Journal of Biological Rhythms, 20, 375-386.

Kuslansky, G. Katz, M., Verghese, J., Hall, C. B., Lapuerta, P. LaRuffa, G., \& Lipton, R. B. (2004). Detecting dementia with the Hopkins Verbal Learning Test and the Mini-Mental State Examination. Archives of Clinical Neuropsychology, 19, 89-104.

Lacerda, S. S., \& Haase, V. G. (2000). Exame neuropsicológico da memória de reconhecimento e da memória de recenticidade. In V. G. Haase, R. Rothe-Neves, M. L. Käppler, M. Teodoro \& G. M. O. Wood (Eds.), Psicologia do desenvolvimento: Contribuições interdisciplinares (pp. 161-178). Belo Horizonte, MG: Health.

Lindeboom, J., \& Weinstein, H. (2004). Neuropsychology of cognitive ageing, minimal cognitive impairment, Alzheimer's disease, and vascular cognitive impairment. European Journal of Pharmacology, 490, 83-86.

May, C. P., Hasher, L., \& Foong, N. (2005). Implicit Memory, age, and time of day Psychological Science, 16, 96-100. 
Monk, T. H. (1994) Chronobiology of mental performance. In Y. Touitou \& E. Raouf (Eds.), Biology rhythms in clinical laboratorie medicine (pp. 208-213). New York: Springer-Verlag.

Nyenhuis, D. L., \& Gorelick, P. B. (1998). Vascular dementia: a contemporary review of epidemiology, diagnosis, prevention and treatment. Journal of American Geriatrics Society, 46, 14371448.

Pompéia, S., \& Bueno, O. F. A. (1998). Preliminary adaptation into Portuguese of a standardized picture set for the use in research and neuropsychological assessment. Arquivos de Neuropsiquiatria, 56, 366-374.

Pompéia, S., Miranda, M. C., \& Bueno, O. F. A. (2001). A set of 400 pictures standardized for portuguese: Norms for name agreement, familiarity and visual complexity for children and adults. Arquivos de Neuropsiquiatria, 59, 330-337.

Rotemberg, L., Portela, L. F., Marcondes, W. B., Moreno, C., \& Nascimento, C. P. (2001). Gênero e trabalho noturno: Sono, cotidiano e vivências de quem troca a noite pelo dia. Cadernos de Saúde Pública, 17, 639-649.

Sachdev, P. S., Brodaty, H., Valenzuela, M.J., Lorentz, L., Looi, J. C. L., Wen, W., \& Zagami, A. S. (2004). The neuropsychological profile of vascular cognitive impairment in stroke and TIA patients. Neurology, 62, 912-919.
Smolensk, M. H. (1998). Knowledge and attitudes of American Physicians and Public about medical chronobiology and chronotherapeutics: Findings of two 1996 gallup surveys. Chronobiology International, 15, 377-394.

Stenberg, G. S., Radeborg, K., \& Hedman, L. R. (1995). The picture superiority effect in implicit conceptual memory tests. Journal of Experimental Psychology: Learning, Memory and Cognition, 23, 425-441.

Wright, K. P., Hull, J. T., \& Czeisler, C. A. (2002). Relationship between alertness, performance, and body temperature in humans. American Journal Physiology Regulatory and Comparative Physiology, 283, R1370-R1377.

Xavier, F. M. F., Ferraz, M. P. T., Pertollucci, P., Poyares, D., \& Moriguchi, E. H. (2001). Episódio depressivo maior, prevalência e impacto sobre qualidade de vida, sono e cognição em octogenários. Revista Brasileira de Psiquiatria, 23, 62-70.

\section{Anexo - Procedimentos de realização dos testes de memória}

- Teste de memória episódica com reconhecimento imediato de figuras (FIGIM): o experimentador apresentava 10 cartões com figuras de objetos e animais (exemplo abaixo), na freqüência de uma por segundo. Em seguida, as 10 figuras foram misturadas a 10 novas figuras e dispostas em cima da mesa. O indivíduo procurava e indicava quais as 10 figuras que tinham sido apresentadas anteriormente.
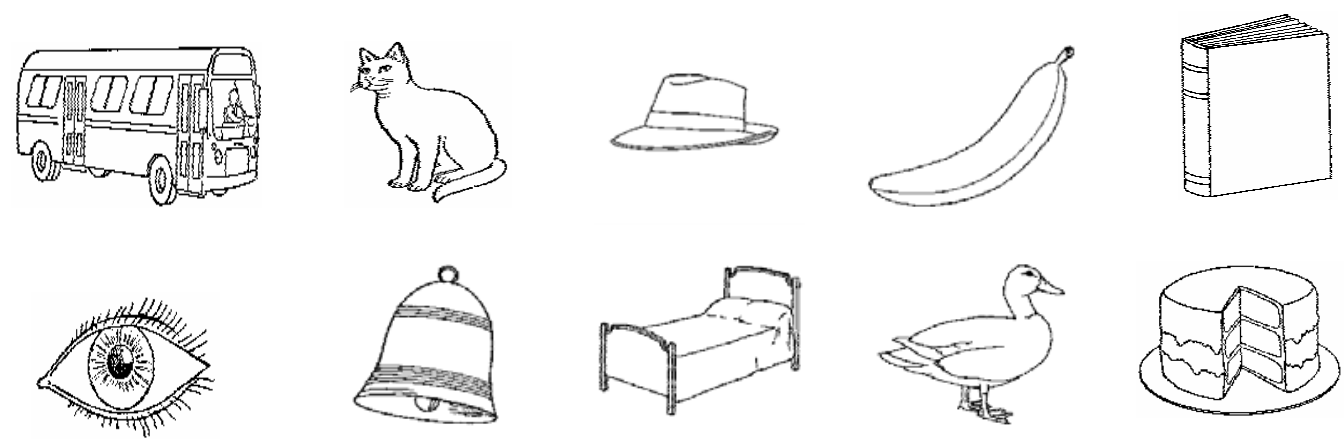

- Teste de memória episódica com evocação livre imediata de palavras (PALIM): consistia na apresentação de uma seqüência de 10 palavras, na cadência de 1 por segundo, onde o indivíduo deveria repetir imediatamente e de forma aleatória (exemplo abaixo).

\begin{tabular}{|l|l|l|l|l|l|l|l|l|l|}
\hline GARRAFA & VELA & FACA & CADEIRA & PENTE & LEÃO & VACA & MARTELO & VESTIDO & MAÇÃ \\
\hline
\end{tabular}

- Teste de memória episódica com reconhecimento tardio de figuras (FIGTAR): o experimentador dispunha 20 cartões com figuras em cima da mesa e o examinando procurava e indicava as 10 figuras que tinham sido apresentadas anteriormente, no início da sessão de testes.

- Teste de memória episódica com reconhecimento tardio de palavras (PALTAR): foi apresentada uma seqüência de 20 palavras onde o indivíduo respondia sim, se a palavra tinha sido apresentada anteriormente, ou não, quando a palavra era desconhecida. 